\section{Bv8: main pain player}

\section{By Michael J. Haas, Senior Writer}

While Roche's Genentech Inc. unit has been busy unraveling the role of Bv8 in tumor angiogenesis, ${ }^{1-3}$ a team of Italian researchers has shown that the target also plays an essential role in inflammatory pain. Local and systemic inhibition of Bv8 signaling treated pain in mice, ${ }^{4}$ potentially providing a route to a new class of therapies.

Despite the recent attention to Bv8 as a cancer target, evidence for its role in the pain setting has been mounting for the past decade. In 1999, a team at the Austrian Academy of Sciences showed that prokineticin 2 (PROK2; Bv8) induced hyperalgesia in rats. ${ }^{5}$ Signaling between the mammalian homolog of Bv8 and its receptors-prokineticin receptor 1 (PROKR1; PKR1) and PROKR2 (PKR2)-has also been implicated in hyperalgesic responses to thermal, mechanical and chemical stimuli. ${ }^{6-8}$

\section{"Maybe Bv8 is one of the main molecules - not just another molecule in the inflammatory soup."}

-Mark Zylka,

The University of North Carolina

at Chapel Hill
Team leader Lucia Negri, professor of physiology and pharmacology at Sapienza, was a member of the 1999 Austrian team that first identified Bv8.

\section{Slicing the space}

are multiple potential options for blocking Bv8 to treat inflampain, including topical or i.v. formulations, and acute or best route is topical therapeutics for acute pain.

Steve Andrews, associate director of drug discovery at Array BioPharma Inc., said the results published in PNAS suggested that topical PKR antagonists could treat lower back pain, gout, surgical pain and osteoarthritis (OA) pain. Compared with systemic therapeutics, he said topical therapies would not have to get into the brain and thus should have fewer CNS side effects. "The challenge of crossing the blood brain barrier has knocked down a lot of pain strategies," he noted.

Array develops small molecules for pain, cancer, inflammation and metabolic diseases.

"The way we think about valid pain targets is that therapies targeting them must have advantages in both efficacy and side effects" relative to existing treatments, said Andrews. "Having one advantage just doesn't get you there."

Although the side-effect profile of molecules that interfere with Bv8-PKR signaling has yet to be explored, Andrews thinks the efficacy of the molecule in the PNAS article "seems compa-

Macrophages, granulocytes and other leukocytes are known to express Bv8, and inflamed tissues overexpress Bv8 mRNA. Nevertheless, the precise role of Bv8 in inflammatory pain has been poorly understood. Thus, researchers at Sapienza University of Rome and colleagues set out to determine whether Bv8 released by tissue-infiltrating leukocytes was an actual trigger of inflammatory pain.

The group found that Bv8 expression was higher in the paws of rodent models of inflammatory pain and hyperalgesia than in those of $P k r 1$ or $P k r 2$ knockouts or wild-type controls. Mice with peritonitis also had higher levels of circulating Bv8-expressing granulocytes and greater paw hyperalgesia than controls.

Local or systemic injections of a nonselective prokineticin receptor (PKR) antagonist previously synthesized by the team ${ }^{9}$ reduced hyperalgesia in both the paw and peritonitis mouse models compared with that in controls.

Additional studies showed that mice deficient in either Pkr1 or $\mathrm{Pkr} 2$ had less paw inflammation than wild-type controls, but only Pkr1 played a role in regulating Bv8 expression.

In a paper in the Proceedings of the National Academy of Sciences, the team wrote that their results suggested a fundamental role of Bv8-PKR receptor signaling in inflammatory pain and that inhibiting the cascade could "constitute a promising strategy for a therapeutic approach to controlling inflammatory pain."

The group also included researchers from the University of Cagliari, the University of Ferrara and the University of Milan. rable to that of NSAIDs."

Mark Zylka, assistant professor of cell and molecular physiology at The University of North Carolina at Chapel Hill's School of Medicine, suggested that an inhibitor of Bv8-PKR signaling could be delivered via a dermal patch. The question, however, is whether the molecule "will reach the source of the pain," he said. "Inflammatory pain arises from sensitization of nerve endings within joints," so it is not clear whether a patch-delivered therapy could reach those nerves.

$\mathrm{Ru}$-Rong Ji, associate professor of anesthesiology at Brigham and Women's Hospital, did not think Bv8 or PKR antagonists were likely to have much utility outside the acute inflammatory pain setting. "Bv8 is produced by neutrophils that only contribute to acute inflammation," he said. "The impact of Bv8/PKR on chronic inflammation and chronic pain-such as neuropathic pain-could be limited."

Zylka agreed that more work is needed to determine whether such antagonists could treat neuropathic and other chronic pain indications. "The Negri team gave the antagonist at 6 hours and 24 hours after the onset in inflammatory pain. They didn't go longer to see whether desensitization to the antagonist occurs with repeated use," he noted.

Andrews said the pathway should be studied in other pain models, such as gout and OA pain, and he wanted to see more evidence that upregulation of Bv8 signaling occurs in human pain. "There are surprisingly few data in the literature about this," he noted.

Ji was less convinced that future studies of the pathway are worth 


\section{TARGETS \& MECHANISMS}

pursuing in pain. "We already have a long list of proinflammatory cytokines and chemokines that can contribute to inflammatory pain sensitization," he said. "Adding another one, Bv8, to the list does not significantly advance our understanding of inflammatory pain.”

Zylka disagreed, noting that the assumption that a host of proinflammatory mediators are involved in inflammatory pain has not been tested. Furthermore, the antagonist described in the PNAS article reduced pain without blocking other proposed proinflammatory mediators, such as bradykinin and prostaglandin $\mathrm{E}_{2}$ $\left(\mathrm{PGE}_{2}\right)$. This finding raises the question of how many of those mediators are actually involved in inflammatory pain, he said.

"Maybe Bv8 is one of the main molecules-not just another molecule in the inflammatory soup," he said. "This paper-and the collective research done by this team-is really impressive, as far as showing the pain-causing effects of Bv8 and the effects of receptor knockout and antagonism. It's worth following up on this with future studies."

\section{Side (effect) show}

Because Bv8 signaling plays roles in neurogenesis, circadian rhythms, angiogenesis and cardiomyocyte function, both Andrews and Zylka think future studies of pathway inhibitors should seek to flesh out side-effect profiles.

"Any pathway that potentially affects cardiovascular function needs to be scrutinized," Andrews said.

He also noted that the Italian researchers found that $P k r 1$ and $P k r 2$ knockout in mice decreased the normal responses to painful thermal and mechanical stimuli. "You don't want a painkiller to do this," he said. "If we saw data that showed inhibition of Bv8 signaling decreased normal pain responses, it would be a show-stopper."

If antagonizing Bv8-PKR signaling does cause neurological or cardiovascular side effects, Zylka said, the question is how they compare with those of existing pain drugs.
According to World Intellectual Property Organization (WIPO) patent records, Sapienza has patented Bv8-derived peptide antagonists of PKR1 and PKR2 to treat pain.

The Negri team was unavailable for comment, and the patent status of the findings reported in PNAS wasn't available.

Genentech has anti-Bv8 antibodies in preclinical development to treat tumors that are resistant to anti-VEGF therapies. The company, which has not disclosed a development timeline for its antiBv8 program, ${ }^{10}$ did not return calls seeking comment on the PNAS paper.

Haas, M.J. SciBX 2(34); doi:10.1038/scibx.2009.1299

Published online Sept. 3, 2009

\section{REFERENCES}

1. Shojael, F. et al. Nature 450, 825-831 (2007)

2. Shojael, F. et al. Proc. Natl. Acad. Sci. USA 105, 2640-2645 (2008)

3. Zhong, C. et al. Clin. Cancer Res. 15, 2675-2684 (2009)

4. Giannini, E. et al. Proc. Natl. Acad. Sci. USA; published online Aug. 10, 2009; doi:10.1073/pnas.0903720106 Contact: Lucia Negri, Sapienza University of Rome, Rome, Italy e-mail: lucia.negri@uniroma1.it

5. Mollay, C. et al. Eur. J. Pharmacol. 374, 189-196 (1999)

6. Negri, L. et al. Br. J. Pharmacol. 137, 1147-1154 (2002)

7. Vellani, V. et al. J. Neurosci. 26, 5109-5116 (2006)

8. Negri, L. et al. J. Neurosci. 26, 6716-6727 (2006)

9. Balboni, G. et al. J. Med. Chem. 51, 7635-7639 (2008)

10. Mikulski, M. SciBX 1(2); doi:10.1038/scibx.2008.29

COMPANIES AND INSTITUTIONS MENTIONED

Array BioPharma Inc. (NASDAQ:ARRY), Boulder, Colo. Austrian Academy of Sciences, Salzburg, Austria Brigham and Women's Hospital, Boston, Mass. Genentech Inc., South San Francisco, Calif. Roche (SIX:ROG; OTCQX:RHHBY), Basel, Switzerland Sapienza University of Rome, Rome, Italy

University of Cagliari, Cagliari, Italy

University of Ferrara, Ferrara, Italy

University of Milan, Milan, Italy

The University of North Carolina at Chapel Hill, Chapel Hill, N.C. 